\title{
Healthcare professional experiences with patients who participate in multimodal pain rehabilitation in primary care - a qualitative study
}

Gunilla Stenberg, Elisabeth Pietila Holmner, Britt-Marie Stalnacke and Paul Enthoven

\author{
Journal Article
}

\section{Tweet}

N.B.: When citing this work, cite the original article.

This is an electronic version of an article published in:

Gunilla Stenberg, Elisabeth Pietila Holmner, Britt-Marie Stalnacke and Paul Enthoven, Healthcare professional experiences with patients who participate in multimodal pain rehabilitation in primary care - a qualitative study, Disability and Rehabilitation, 2016. 38(2021), pp.2085-2094.

Disability and Rehabilitation is available online at informaworldTM:

http://dx.doi.org/10.3109/09638288.2015.1114156

Copyright: Taylor \& Francis: STM, Behavioural Science and Public Health Titles http://www.tandf.co.uk/journals/default.asp

Postprint available at: Linköping University Electronic Press

http://urn.kb.se/resolve?urn=urn:nbn:se:liu:diva-131723

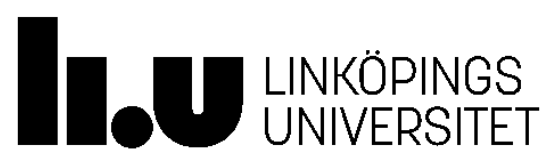


Healthcare professional experiences with patients who participate in multimodal pain rehabilitation in primary care - a qualitative study

Gunilla Stenberg${ }^{1}$, Elisabeth Pietilä Holmner ${ }^{2}$, Britt-Marie Stålnacke², Paul Enthoven ${ }^{3}$

${ }^{1}$ Department of Community Medicine and Rehabilitation, Physiotherapy, Umeå University, SE-901 85 Umeå, Sweden

${ }^{2}$ Department of Community Medicine and Rehabilitation, Rehabilitation Medicine, Umeå University, SE-901 85 Umeå, Sweden

${ }^{3}$ Department of Medical and Health Sciences, Linköping University SE-581 85 Linköping, Sweden

\begin{abstract}
Purpose

Exploring healthcare professional experiences of Multimodal rehabilitation (MMR) in primary care.

Methods
\end{abstract}

Fourteen healthcare professionals (11 women, 3 men) were individually interviewed about their work with MMR in primary care. Interviews covered experiences of assessing patients and work with patients in the program. Transcribed interviews were analysed by qualitative content analysis.

Results

The analysis resulted in four categories: select patients for success; a multilevel challenge; ethical dilemmas; and considering what is a good result. MMR work was experienced as useful and efficient, but also challenging because of patient complexity. Preconceptions about who is a suitable patient for MMR influenced the selection of patients (e.g., gender, different culture). Interviewees were conflicted about not to being able to offer MMR to patients who 
were not going to return to work. They thought that there were more factors to evaluate MMR than by the proportion that return to work.

\section{Conclusions}

Healthcare professionals perceive MMR as a helpful method for treating chronic pain patients. At the same time, they thought that only including patients who would return to work conflicted with their ethical views on equal healthcare for all patients. Preconceptions can influence selection for, and work with, MMR.

Keywords: multimodal treatment, pain, immigrants, gender bias, health care professionals

\section{Introduction}

Chronic musculoskeletal pain is a major problem in most industrialized countries, with a prevalence of around 35\% in the general population and affecting more women than men [1,2]. In Sweden, musculoskeletal pain is one of the most common reasons for a primary care visit, with $20-40 \%$ of those who seek care suffering from pain conditions [3,4].

Individuals with chronic pain generally describe wide-ranging consequences, including psychological distress, insomnia, reduced work ability/sick leave, and low quality of life [59]. For society, chronic pain has major consequences and high economic costs. In Sweden during 2009, the cost of musculoskeletal disorders amounted to SEK 20 billion, and represented $31 \%$ of the total health insurance costs [10]. Thus, sustainable treatment effects are needed for chronic pain patients. Previous studies have shown that multimodal rehabilitation (MMR) is efficacious in increasing activity levels and reducing sick leave [11,12]. MMR is based on a biopsychosocial model of chronic pain that considers somatic, psychological, environmental, and personal aspects [13]. MMR can involve several professionals who work together in a team that includes the patient as a member and includes 
a combination of physical and physiological interventions over a lengthy time period, with a common goal for the individual patient.

MMR has primarily been provided by healthcare specialists for patients with complex chronic pain. However, as consequence of the 2008 Swedish national rehabilitation warranty, MMR programs were implemented in primary healthcare for patients with less complex chronic pain. This implementation is linked to financial compensation (i.e., a rehabilitation warranty) for each patient who participates in MMR. The primary aims of the rehabilitation warranty are to increase the return to work rate and reduce sickness absence. National guidelines for selection criteria were published to support assessment of patients with chronic pain, and thereby offer MMR on the appropriate level (specialist vs. primary healthcare) [14]. Because MMR programs are now used in primary healthcare in Sweden, this implementation needs to be evaluated. There is also a lack of knowledge about primary healthcare professional perceptions of working with MMR for chronic pain patients. Therefore, we conducted a qualitative study with the aim of exploring healthcare professional experiences of MMR in primary care.

\section{Methods:}

This was a qualitative interview study conducted in primary healthcare in Sweden.

\section{Setting}

Participants were recruited from multimodal rehabilitation teams in primary healthcare in two Swedish county councils, one from the northern part and the other from the southern part of the country. MMR teams consisted of different professionals in different teams but involved at least a physiotherapist and occupational therapist. In each team, general practitioners, social 
workers or psychologists were either part of the team or had a consulting role (Table 1). In teams that consisted only of a physiotherapist and an occupational therapist, at least one of them was trained in cognitive behavioural therapy. For background data see Tables 1 and 2. Healthcare professionals at primary healthcare centres referred patients to the MMR teams for MMR, and the MMR team assessed and selected patients appropriate for rehabilitation. MMR was conducted as a group, individually, or in a combination of group and individual components. Inclusion criteria for MMR were age 18-65 years, disabling chronic pain, potential for an active life change, and no other disease or other state that precluded participation in the program. Patients needed to be on sick leave, or experiencing major interference in daily life due to chronic pain and thus at risk of ending up on sick leave.

\section{Insert table 1 about here}

\section{Subjects and data collection}

All healthcare professionals who worked with MMR in the two county councils were informed about the study by email or telephone, and invited to participate. The initial invitation resulted in five participants. After that, a purposive sample was chosen to provide variability in age, profession, extent of experience, team size, and patient catchment area. Fourteen healthcare professionals (eleven women and three men) from 10 of 11 multimodal teams volunteered. This was judged to be a large enough sample to provide a variety of experiences and to allow sufficient depth in the analysis. Additional pertinent background data are provided in Table 2. All interviewees gave written informed consent prior to the interviews. 
Insert table 2 about here

An interview guide was developed by the research group and used to remind the interviewer of topics to include. The guide covered benefits and drawbacks of including patients in MMR, the types of patients in MMR, factors that facilitate or impede conduct of MMR, professional views on MMR, and teamwork experiences. All interviews began with the open-ended question, “What are your experiences of working with multimodal rehabilitation?”

The first author (GS) conducted the interviews, which occurred between August 2013 and January 2014. Interviews were conducted at the interviewee’s workplace, and lasted 40-70 minutes. They were audiotaped and transcribed verbatim.

\section{Analyses}

We organized the data into two content areas: occupational perspectives of starting and working in a multimodal team (analysis underway), and experiences among healthcare professionals when assessing chronic pain patients and working with them in multimodal pain rehabilitation. A content area is an elementary structure of content that can be recognized with little interpretation and demarcate specific topics in the material [15].

The interviews were analysed by qualitative content analysis [15], which is a systematic method useful when analysing data on people’s experiences and reflexions [16]. The analysis started with reading interviews to get an overview of the entire text. Next, the coding process began with division of the text into meaning units. Each meaning unit was labelled with a code. The coding process was done with OpenCode 4.0 Umeå software (Umeå University; 
2013 ICT Services and System Development and Division of Epidemiology and Global Health) (2013).

The first author (GS) coded the entire material, and two authors (EPH, PE) each coded three interviews for triangulation. The codes were sorted and grouped into subcategories and categories during discussion and negotiation between all authors. Table 3 gives an example of the coding process. During the analysis, the first author made field notes of reflections and interpretations. To enhance reflexivity and trustworthiness, the results were presented and discussed in two seminars: one with qualitative researchers from research fields other than chronic pain, and one with different healthcare professionals.

This study was approved by the ethics committee of the Medical Faculty of Umeå University (Dnr 2013-192-31 M).

Insert table 3 about here

\section{Results}

The analysis resulted in four categories: 1) select patients for success, 2) a multilevel challenge, 3) ethical dilemmas, and 4), considering what is a good result (Table 4). Interviewees described MMR with joy and enthusiasm, but also noted the challenges and difficulties of working with chronic pain patients in this way. The emergent categories are presented below, with quotations in italics. 


\section{Select patients for success}

The process of selecting patients for MMR in primary healthcare was viewed differently among interviewees, and varied according to the availability of appropriate patients. Some used diagnoses or questionnaires; others thought that tools were inadequate to identify appropriate patients. Some MMR teams found it difficult to identify appropriate patients according to the guidelines, and some teams were forced to include all referred patients to have enough participants for a group. Others could select the patients who they thought might benefit from MMR, and they described how they became skilled over time at identifying successful patients.

"I think that we've tried to include patients who we thought are in a phase where they are able to make life changes, and most (of the patients) have jobs....So, it is very few who are unemployed that I can recall....I think that, on the whole, it has been the right patients in the MMR group.”

Interviewees reported that high consumers of healthcare, and patients suffering with chronic pain are more difficult to rehabilitate and return to work. Many of the interviewees said they were surprised when patients who they thought impossible to rehabilitate, started life changes. "What we noticed...is that we can't say beforehand who actually manages to motivate her/himself and recover, because sometimes we... have a feeling that (for) some people this might not work. But sometimes it does."

Participation only in group treatment was recommended to most patients. Some were recommended for group and individual treatment, but there were also a few patients who only 
received individual treatment. Reasons for individual treatment alone were that the patient was not felt likely to be successful in a group (e.g., did not like to talk in group settings), was a non-Swedish speaker, had individual needs, or had low motivation.

Selection of patients was sometimes based on previous experiences of group dropouts. Patients declined MMR participation because they were unable to leave work, had long travel distances, or difficulty understanding the value of MMR. Among patients invited to participate, more men than women were hesitant to join the programs but men who participated were often satisfied.

The importance of information exchange and good communication between the team and referring healthcare professionals was emphasized. At primary healthcare centres with only a few referred patients, it was thought that this could be due to preconceptions about which patients could benefit. A higher proportion of women than men was referred, and later selected for MMR group treatment. Interviewees thought that this was due to different views on women and men; men were more often perceived to have real pain with clear causes, and therefore men were easier to investigate and refer to specialists. Men were rarely thought to fit the current picture of a MMR patient.

"It is...few men who have generalized pain like fibromyalgia, etc. It may be more common among men to have back pain, hip pain, osteoarthritis of the knees and so on, and be waiting for orthopaedic consultations etc., and they're supposed to have completed investigations (to participate in MMR). So those who come (for care), they might be, for example, those who have residual symptoms with headache or something similar...perhaps many years after a traffic accident. They manage themselves pretty well, but still need to learn to deal with 
(pain). It might also be that they have had pretty tough jobs, which caused them to function inadequately."

Some of the interviewees wondered whether it was always the actual patient needs that determined whether they would be offered MMR. Perhaps preconceptions about men and women's behaviours were the determining factor. Women are viewed as more likely to participate in group treatment, more used to talking and working in groups, and that it is more acceptable for women to talk about feelings. Some interviewees did not offer MMR group treatment to men unless it was possible to have at least two men in the group. They thought that one man could be lonely in a group of women. Women and men were thought to express themselves in different ways. Such interpretations by healthcare professionals could result in men not having the opportunity to participate in MMR.

"I think that patients (women and men) express themselves differently when seeking care. And the person who receives those words chooses differently based on that."

There were different opinions among the interviewees about whether male professionals on the team resulted in an increased number of men in MMR, and there were few men working on the MMR teams.

Different approaches existed toward patients who did not speak Swedish. These patients were primarily immigrants.

Some were offered individual treatment; others were offered group treatment with an interpreter for immigrant patients (only); still others did not think immigrants were potential MMR patients.

"So far we have not had someone like that (immigrant)... but...I have had patients with an interpreter who I haven't referred to MMR....perhaps I have not explicitly thought that it 
won't work with MMR, but there are a lot of non-Swedish speaking patients seeking care for chronic pain. And maybe...they will be taken care of in other ways. It becomes individual treatment, but not MMR. "

Doubts regarding appropriateness of group treatment for immigrants were often related to the need for an interpreter, and different views on pain in different cultures. These factors were thought to have a negative impact on the MMR group process, and such patients were only offered individual MMR or no MMR at all. For example, some interviewees thought that it was important to first find out an individual patient's thoughts about pain, as a sort of calibration, and then to start from there.

\section{A multilevel challenge}

Most of the interviewees experienced MMR treatment of chronic pain patients as a challenge on several levels. Work in chronic pain was described as hard when dealing with patients whose experience was that they had tried everything in healthcare, who were not self-driven, and who were in need of much support. Patients could be perceived as demanding the way that they wanted to get a diagnosis and wanted to be cured. In general, healthcare professionals were described as having difficulty setting limits and stopping an investigation because of fear of missing a serious illness. Immigrants with chronic pain were viewed to be especially challenging, sometimes because of language difficulty, and sometimes because of their traumatic experiences.

Some of the interviewees saw a challenge in the fact that more women than men were referred to MMR. Some were up to date with the latest research on gender medicine; others said that they were not at all interested (in gender medicine). 
From a gender perspective, group treatment could be experienced as a challenge because it was easy to get into gender-stereotype discussions in the groups when only women were represented. Others thought that when treatment is adjusted to group members, MMR is gender neutral, and they no longer need to take gender into account.

Discussions about inequity were common in the MMR groups, but interviewees felt that it was hard to raise questions about violence and threats if there were men in the group. These topics were easier to talk about during individual treatment. Another challenge was that when men were present (mixed groups), women were sometimes less open during discussions and had other roles, e.g., being more caring (about the men).

Interviewees who had experience with men in MMR groups mostly told about their positive experiences. Men were often satisfied with their treatment. At times, a man was the only man in the group. Interviewees said that discussions were more open and straightforward in groups with both women and men.

In light of these challenges, interviewees had positive experiences from working with MMR that they thought could be very helpful.

"For me, it is primarily a nice way to work with this group of patients. They are difficult patients who are complicated, and it is very hard to work alone with these complex problems." 
The interviewees described how complex patients could sometimes be perceived as a positive challenge. To work against the odds and try to find individual solutions was stimulating.

"I think there must be an interest (for the MMR team member) in working with something that is complex. I think so. To help those people who others (healthcare professionals) do not bother to help or do not want to help. I think someone has to help them (patients). I want to be the one who helps those who are most 'in the shit', if one may say so.... I like when it's difficult."

\section{Ethical dilemmas}

The guidelines and rehabilitation warranty were intended at getting chronic pain patients to stay at work or return to work after sick leave. Those interviewed felt that their duty to the healthcare legislation (aspects of equal care for all patients) conflicted with the MMR guidelines if they could not offer MMR to all chronic pain patients. This was especially the case for patients with the greatest need of rehabilitation-e.g., individuals who did not speak Swedish, or elderly persons who did not fit the inclusion criteria used for returning to work. This led to frustration among some of the interviewees. The interviewees wanted other, additional, aims for MMR, such as less suffering or better quality of life.

"....(By offering this treatment), we have said to people what their life should be about; (we) say that to have (good) quality of life, one should work. For me it's incredibly frustrating because somehow we go into some kind of judgmental, inflexible attitude toward the patient that makes it much harder to meet them where they are, and they have already been...rejected by many healthcare providers. And we start by telling them what their life should be about. 
For me it would be much better if the rehab warranty was about improving quality of life (not just returning to work). "

Some interviewees disregarded the rules and offered rehabilitation to patients who would have been excluded because of the requirement to return to work, e.g., patients on long-term sick leave.

Considering what is a good result Interviewees experienced ambivalent feelings about MMR results. Some deliberated on the value of MMR and discussed the results in relation to costs and the effort they put into the treatments. These comments were especially related to the many dropouts and the difficulty of knowing beforehand who could be a successful patient.

"... It (MMR) takes a lot of time, lots of resources, (of) energy. Sometimes you might think, well what benefit do you have in the end, really? Because there's a lot that we can't have an effect on."

However, teamwork was reported to make MMR effective. Teamwork gives different perspectives on assessment and rehabilitation. Some of the interviewees were frustrated by the short, allowable MMR period of 6-8 weeks. They felt that it was too short of a time to result in behavioural change. They also emphasized that a longer follow-up period of at least 2 years is needed to capture life changes.

On the other hand, most interviewees thought that patients were satisfied and benefit from MMR. For example, among those patients who completed the MMR there was less healthcare 
consumption, better coping with pain, and patients returned to work or started to study. MMR led to larger or smaller life changes, but this was not always seen in the follow-up questionnaires. Interviewees thought that, at least from the patient view, there are more factors and different ways to evaluate MMR than by the proportion that return to work. Study of health-related quality of life and lower healthcare consumption were two suggested factors.

"A problem is that we only look at each individual organization. But if we look at both the patient's suffering, and that the patient gets a better quality of life, this ought to be the main reason why we treat in this way. We can also look in a longer perspective, which is to say if people actually manage to work again, we have a tremendous profit. If we only get one person back to work...then it's a tremendous profit. I think many times we forget to look at the long run. In addition, we also may have persons who do not seek care for long(er) periods because they can handle this better themselves (after MMR). So there are several parts of this, like a societal perspective and a person's suffering. There is much to gain.”

\section{Discussion}

Interviewees thought MMR was a useful intervention for chronic pain patients, but also challenging in many ways. Despite national criteria, selecting patients for MMR in primary health care could be difficult and was influenced by preconceptions of patients such as gender and culture. Identifying which patient could benefit from MMR before starting was difficult. Patients were sometimes perceived as demanding; this was not entirely negative, but could also be stimulating. The interviewees felt that their duty to comply with healthcare legislation conflicted with guidelines about returning to work. 
Although there were national guidelines, selection criteria differed among the MMR teams. Some units strictly adhered to the national guidelines, and some trusted their long experience when selecting patients. Even patients on long-term sick leave could be selected because they were assessed as motivated and should benefit by improving quality of life, or more patients were needed for group treatment. Hellman et al reported similar findings [17]. Those interviewed in the Hellman study justified inclusion of patients on long-term sick leave by concluding that health and wellbeing improved even if patients did not return to work. These benefits may impact rehabilitation outcomes and could be considered when evaluating MMR as a rehabilitation method.

Men were offered MMR less often than women in our study. According to the interviewees, this could be due to differences in how men and women talk about their pain. The way a patient views himself or herself is important in the communication between patient and healthcare professionals, and can influence decision-making. Studies show that women and men with the same symptoms present them in different ways [18-20]. Healthcare professionals might not perceive real needs that are hidden behind gender stereotypes [21]. As in our study, O’Brien et al [22] and Ahlsen et al [21] found that men’s health-seeking behaviours were due to visible, physical symptoms. In our study, interviewees thought that men were more often given a diagnosis other than unspecified chronic pain. Other studies have shown that non-specific diagnoses are more often assigned to women than to men $[19,23]$. This could be because healthcare professionals are not comfortable giving men a diagnosis that is more common among women. Ahlsen et al [21] saw this in her study, when healthcare professionals working with chronic pain patients were hesitant to give men a diagnosis associated with a woman's disease, e.g., fibromyalgia, because they thought men would feel unsupported with this diagnosis. 
In our study, being an immigrant could hinder selection for MMR in primary healthcare. In a review on ethnic disparities in pain, Andersson et al [24] found that gender and ethnicity predicted the amount and type of treatment for chronic pain patients, with least support for non-majority women. Sethi [25] stated the importance of knowledge among healthcare personnel about problems related to immigration, and the need to be familiar with the situation for immigrants in their new setting. This can have an impact on pain experience and also how immigrants view and manage pain [25].

In a study by Hammarstrom et al [26], low-educated women were offered MMR less often by specialists. This was not reported in our study, but in the future, rehabilitation team members as well as rehabilitation actors on organizational levels (e.g., managers policy makers) must be more aware of how the intersection of gender, ethnicity, social class, and sexuality contribute to how patient needs are viewed and who is offered rehabilitation. These types of factors interact and can contribute to further needs [27].

Interviewees in the current study saw challenges in their work with MMR. For example, they thought language barriers, different views on pain, and how patients from other cultures expressed pain, could be problems in rehabilitation. This was also reported in a UK study by Patel et al [28]. However, cultural differences in expression of pain exist between cultures as well as within a culture [29]. Other factors such as socioeconomic conditions [30] and gender $[18,29]$ can also be important for expression of pain and should be considered, but not hinder, rehabilitation. 
Another idea expressed by the interviewees was that men did not fit into group treatment because they could not talk about feelings. There is a risk that healthcare professionals do not feel comfortable with posing questions about emotions to men. Therefore, they do not ask such questions [21] or are unwilling to listen empathically to men's experiences [31]. This might be the experience of those interviewed in our study, and could be due to the cultural role of gender in men's presentation of chronic pain [21].

Some of the interviewees in our study found it difficult to handle both women and men in same group. There is some support for gender sensitive groups in other patient groups [32]. This needs to be further studied in group rehabilitation of chronic pain patients. However, interviewees in our study who had men and women together in group rehabilitation had good experiences, even if it was a challenge to handle questions about equality and abuse.

The interviewees perceived that the MMR work was sometimes in conflict with their ethical views on equal healthcare for all patients. The Swedish healthcare system is largely taxpayer funded. By law, everyone in Sweden has the right to obtain the care that his/her needs require [33]. Care is to be provided with respect for the equal worth of all people, and the dignity of the individual. Priority is to be given to those who are in the greatest need, and healthcare should be reasonably cost-effective. Swedish healthcare workers are responsible for how they carry out their duties and must perform their work in line with science and best practices [34]. According to Swedish health authorities, there is a medical indication for MMR if the patient has chronic pain if this limits the patient's daily life to a high degree, and if the patient has the potential to improve despite the pain [14]. This means that even patients who do not belong to the guideline target group might benefit from MMR. The interviewees were squeezed between the rules set up by the rehabilitation warranty and other Swedish laws that state that 
all patients should receive the best possible care. According to the rehabilitation warranty, success with MMR is highly connected with work-related outcomes. In contrast, healthcare professionals are usually most interested in health-related outcomes. This was seen in another study when healthcare professionals working with MMR were more focused on psychological and physical well-being, and patient participation in everyday life, than on return to work [17].

Some of our interviewees were frustrated about the short length of the rehabilitation period. In a 2010 review of multimodal rehabilitation by the Swedish Council on Health Technology Assessment, large variation in MMR programs was observed [12]. In contrast, studies have found $[7,35]$ significant improvement in pain and pain-related measures after a two-day team assessment.

\section{Methodological discussion}

A study strength was that interviewees were recruited from different parts of Sweden, and worked at different primary care settings. The interviewees were chosen through purposeful sampling to enhance rich variation in data and to gain credibility [15]. The interviewees had different ages, professions, length of experience, team size, patient catchment areas, and both genders were interviewed. Interview of more male healthcare professionals would have been preferable, but most staff who work with MMR are women. This may have influenced the results. However, answers from the three men in our study did not differ from other answers. When doing interview studies, there is a risk that only interested and positive individuals participate. A strength in our study is that both positive and negative opinions about MMR were presented. 
A shortcoming in this study is that there were no clinical psychologists who participated. This was a clinical study, and clinical psychologists are rare in primary healthcare in Sweden. The national guidelines do not require a psychologist on the team, but require cognitive behavioural competence on all teams. To attain this, team members (social workers, occupational therapists, registered nurses or physiotherapists) had education in cognitive behavioural therapy and how physical, psychological and social factors influence chronic pain.

Having a research group that consists of both women and men, as well as different professions, was a strength during the study analyses. This diversity permitted different perspectives on the findings. We could have involved other professions in the research group to further strengthen the work. Instead, colleagues from other professions and a group of qualitative researchers were asked to reflect on the findings during different stages of the analysis. The input from their comments was considered in the interpretation. Two of the authors have clinical experience from MMR work in a specialty clinic. The others have clinical experience from primary healthcare. Each author tried to respond reflexively to the way their clinical experiences may have impacted the analysis. We could have involved interviewees in the research process to further strengthen the work.

Most interviewees had worked with MMR since introduction of the (national) rehabilitation warranty, but there were two who had worked in primary care MMR for many years prior to establishment of the national guidelines. However their experiences were consistent with the other interviewees. 
When working with the data analysis, information about content of MMR related to the guidelines came up. We chose not to process this because it was in accordance with the guidelines, and no new information came forth.

This study was conducted in Sweden. The same study, performed in a society with a different healthcare system or other norms about chronic pain, gender, or immigrants, might yield different findings.

\section{Conclusions}

Healthcare professionals perceived multimodal rehabilitation (MMR) to be a useful and efficient way to treat chronic pain patients in primary care. The work was felt to be challenging in the selection and treatment of patients. Preconceptions about who are appropriate MMR patients should be dealt with in MMR inclusion criteria. Conflicting emotions exist about inclusion criteria and ethical views on equal healthcare for all patients. Healthcare professionals must be supported in their work with chronic pain patients, and given the opportunity to reflect on their own views of chronic pain patients, gender, culture, and return to work. The results from this study could be valuable when organizing future MMR in primary healthcare.

\section{Acknowledgement}

We would like to thank the healthcare professionals who shared their rich stories.

\section{Declaration of interest}


The authors report no declaration of interest. This study was supported by the Swedish Social Insurance Agency, the research program REHSAM.

\section{References}

1. Bergman S, Herrström P, Högström K, Petersson IF, Svensson B, Jacobsson L. Chronic musculoskeletal pain, prevalence rates, and sociodemographic associations in a Swedish population study. The Journal of rheumatology 2001:1369-1377.

2. Gerdle B, Björk J, Henriksson C, Bengtsson A. Prevalence of current and chronic pain and their influences upon work and healthcare-seeking: a population study. The Journal of Rheumatology 2004:1399-1406.

3. Mäntyselkä P, Kumpusalo E, Ahonen R, Kumpusalo A, Kauhanen J, Viinamäki H, Halonen P, Takala J. Pain as a reason to visit the doctor: a study in Finnish primary health care. Pain 2001:175-180.

4. Hasselström J, Liu-Palmgren J, Rasjö-Wrååk G. Prevalence of pain in general practice. European journal of pain 2002:375-385.

5. Brown JL, Edwards PS, Atchison JW, Lafayette-Lucey A, Wittmer VT, Robinson ME. Defining Patient-Centered, Multidimensional Success Criteria for Treatment of Chronic Spine Pain. Pain Medicine 2008:851-862.

6. Casarett D, Karlawish J, Sankar P, Hirschman K, Asch DA. Designing pain research from the patient's perspective: what trial end points are important to patients with chronic pain? Pain Medicine 2001:309-316.

7. Merrick D, Sundelin G, Stålnacke B-M. One-year follow-up of two different rehabilitation strategies for patients with chronic pain. Journal of rehabilitation medicine 2012:764-773. 
8. Robinson ME, Brown JL, George SZ, Edwards PS, Atchison JW, Hirsh AT, Waxenberg LB, Wittmer V, Fillingim RB. Multidimensional success criteria and expectations for treatment of chronic pain: the patient perspective. Pain Medicine 2005:336-345.

9. Turk DC, Dworkin RH, Revicki D, Harding G, Burke LB, Cella D, Cleeland CS, Cowan P, Farrar JT, Hertz S. Identifying important outcome domains for chronic pain clinical trials: an IMMPACT survey of people with pain. Pain 2008:276-285.

10. Försäkringskassan; Vad kostar olika sjukdomar i sjukförsäkringen? [What are the costs for different diseases in Swedish sickness insurance.] Social Insurance Report; 2011:4 Stockholm (in Swedish)

11. Scascighini L, Toma V, Dober-Spielmann S, Sprott H. Multidisciplinary treatment for chronic pain: a systematic review of interventions and outcomes. Rheumatology 2008:670-678.

12. Statens beredning för medicinsk utvärdering. Rehabilitering vid långvarig smärta en systematisk litteraturöversikt.[ Rehabilitation of patients with chronic pain conditions : a systematic review.] Stockholm: Statens beredning för medicinsk utvärdering (SBU); 2010. (in Swedish).

13. Gatchel RJ, Peng YB, Peters ML, Fuchs PN, Turk DC. The biopsychosocial approach to chronic pain: scientific advances and future directions. Psychological bulletin 2007:581.

14. Nationella medicinska indikationer. Indikationer för multimodal rehabilitering vid långvarig smärta [Indication for multimodal rehabilitation in chronic pain.] Svenska läkaresällskapet, Socialstyrelsen, Sveriges kommuner och landsting, Statens beredning av medicinsk utvärdering. Rapport 2011:2 Stockholm. (in Swedish) 
15. Graneheim UH, Lundman B. Qualitative content analysis in nursing research: concepts, procedures and measures to achieve trustworthiness. Nurse Educ Today 2004:105-12.

16. Downe-Wamboldt B. Content analysis: method, applications, and issues. Health Care Women Int 1992:313-21.

17. Hellman T, Jensen I, Bergström G, Busch H. Returning to work-a long-term process reaching beyond the time frames of multimodal non-specific back pain rehabilitation. Disability \& Rehabilitation 2014:1-7.

18. Stenberg G, Fjellman-Wiklund A, Ahlgren C. "Getting Confirmation”: Gender in Expectations and Experiences of Healthcare for Neck or Back Patients. Journal of rehabilitation medicine 2012:163-171.

19. Ahlgren C, Hammarstrom A. Back to work? Gendered experiences of rehabilitation. Scand J Public Health 2000:88-94.

20. Elderkin-Thompson V, Waitzkin H. Differences in clinical communication by gender. J Gen Intern Med 1999:112-21.

21. Ahlsen B, Mengshoel AM, Solbrække KN. Troubled bodies-troubled men: a narrative analysis of men's stories of chronic muscle pain. Disability and rehabilitation 2012:1765-1773.

22. O’brien R, Hunt K, Hart G. 'It's caveman stuff, but that is to a certain extent how guys still operate': men's accounts of masculinity and help seeking. Social science \& medicine 2005:503-516.

23. Hamberg K, Risberg G, Johansson E, Westman G. Gender bias in physicians' management of neck pain: a study of the answers in a Swedish national examination. $\mathrm{J}$ Womens Health Gend Based Med 2002:653-66. 
24. Anderson KO, Green CR, Payne R. Racial and ethnic disparities in pain: causes and consequences of unequal care. The Journal of Pain 2009:1187-1204.

25. Sethi B. Newcomers health in brantford and the counties of brant, haldimand and Norfolk: perspectives of newcomers and service providers. Journal of Immigrant and Minority Health 2013:925-931.

26. Hammarström A, Haukenes I, Wiklund AF, Lehti A, Wiklund M, Evengård B, Stålnacke B-M. Low-educated women with chronic pain were less often selected to multidisciplinary rehabilitation programs. PloS one 2014:e97134.

27. Crenshaw K. Demarginalization the Intersection of Race and Sex. A Black Feminist Critique of Antidiscrimination Doctrine. Feminist Theory, and Antiracist Politics. In: Ann Philips (Hg.): Feminism and Politics. New York: Oxford University Press 1998:314-342.

28. Patel S, Peacock S, Mckinley R, Clark-Carter D, Watson P. GPs’ perceptions of the service needs of South Asian people with chronic pain A qualitative enquiry. Journal of health psychology 2009:909-918.

29. Nayak S, Shiflett S, Eshun S, Levine F. Culture and gender effects in pain beliefs and the prediction of pain tolerance. Cross-Cultural research 2000:135-151.

30. Ernst G. The Myth of the'Mediterranean Syndrome': Do immigrants feel different pain? Ethnicity and Health 2000:121-126.

31. Sparkes AC, Smith B. Sport, spinal cord injury, embodied masculinities, and the dilemmas of narrative identity. Men and masculinities 2002:258-285.

32. Strömbäck M, Malmgren-Olsson E-B, Wiklund M. 'Girls need to strengthen each other as a group': experiences from a gender-sensitive stress management intervention by youth-friendly Swedish health services-a qualitative study. BMC public health 2013:907. 
33 Sveriges Riksdag Socialdepartementet. Hälso- och sjukvårdslag (1982:763). [The Health and Medical Services Act.] Sveriges riksdag;1982:763. Stockholm (in Swdish)

34. Sveriges Riksdag Socialdepartementet: Lag om yrkesverksamhet på hälso- och sjukvårdens område.[The Act on Professional Activity in Health and Medical Services]. Sveriges Riksdag; 1998:531. Stockholm (in Swedish)

35. Pietilä-Holmner E, Fahlström M, Nordström A. The effects of interdisciplinary team assessment and a rehabilitation program for patients with chronic pain. American Journal of Physical Medicine \& Rehabilitation 2013:77-83. 
Table 1. Background data for the interviewees.

\begin{tabular}{|c|c|c|c|c|c|}
\hline Interviewee & Profession & Sex/age, years & Professional experience (years) & Rural or Urban area & Experiences of multimodal rehabilitation (years) \\
\hline IP1 & OT & $\mathrm{W} / 53$ & 27 & urban & 3.5 \\
\hline IP2 & GP & $\mathrm{W} / 31$ & 1 & urban & 0.7 \\
\hline IP3 & OT & W/55 & 21 & urban & 3 \\
\hline IP4 & PT & $\mathrm{W} / 48$ & 30 & urban & 4 \\
\hline IP5 & PT & $\mathrm{W} / 44$ & 19 & rural & 3.5 \\
\hline IP6 & PT & $\mathrm{W} / 32$ & 7 & urban & 1 \\
\hline IP7 & $\mathrm{RN}$ & W/59 & 12 & rural & 4 \\
\hline IP8 & OT & W/55 & 33 & urban & 17 \\
\hline IP9 & $\mathrm{PT}$ & W/63 & 42 & rural & 7 \\
\hline IP10 & $\mathrm{RD}$ & W/41 & 15 & urban & 2 \\
\hline IP11 & GP & $\mathrm{M} / 60$ & 26 & urban & 1.5 \\
\hline IP12 & PT & $\mathrm{M} / 30$ & 6 & urban & 1 \\
\hline IP13 & $\mathrm{PT}$ & $\mathrm{M} / 51$ & 22 & rural & 5 \\
\hline IP14 & SW & $\mathrm{W} / 43$ & 8 & rural & 3 \\
\hline
\end{tabular}

W: woman, M: man, OT: occupational therapist, GP: general practitioner, PT: physiotherapist, RN: nurse, RD: dietician, SW: social worker. 
Table 2. Subcategories and examples of codes in the category "Putting focus on rehabilitation"

\begin{tabular}{|c|l|l|}
\hline Category & Subcategory & Examples of codes \\
\hline
\end{tabular}

Putting the focus on rehabilitation Other duties easily given priority over rehabilitation Acute problems the focus in primary care

Primary care management does not prioritize multimodal rehabilitation

Low status in working with chronic pain patients

Lack of understanding from colleagues

Patients who can be cured are higher priority

Low status because chronic pain is a women's concern

Health care management needs knowledge

Needs knowledge about professional competencies

A lot of work needed to educate managers

In need of support from healthcare management

Manager supports new ideas

Must find own solutions when manager disinterested

Little understanding of the work load from management 
Table 3. Categories and subcategories important for successful implementation of multimodal rehabilitation in primary care, and the professional perspective on gains from multimodal rehabilitation.

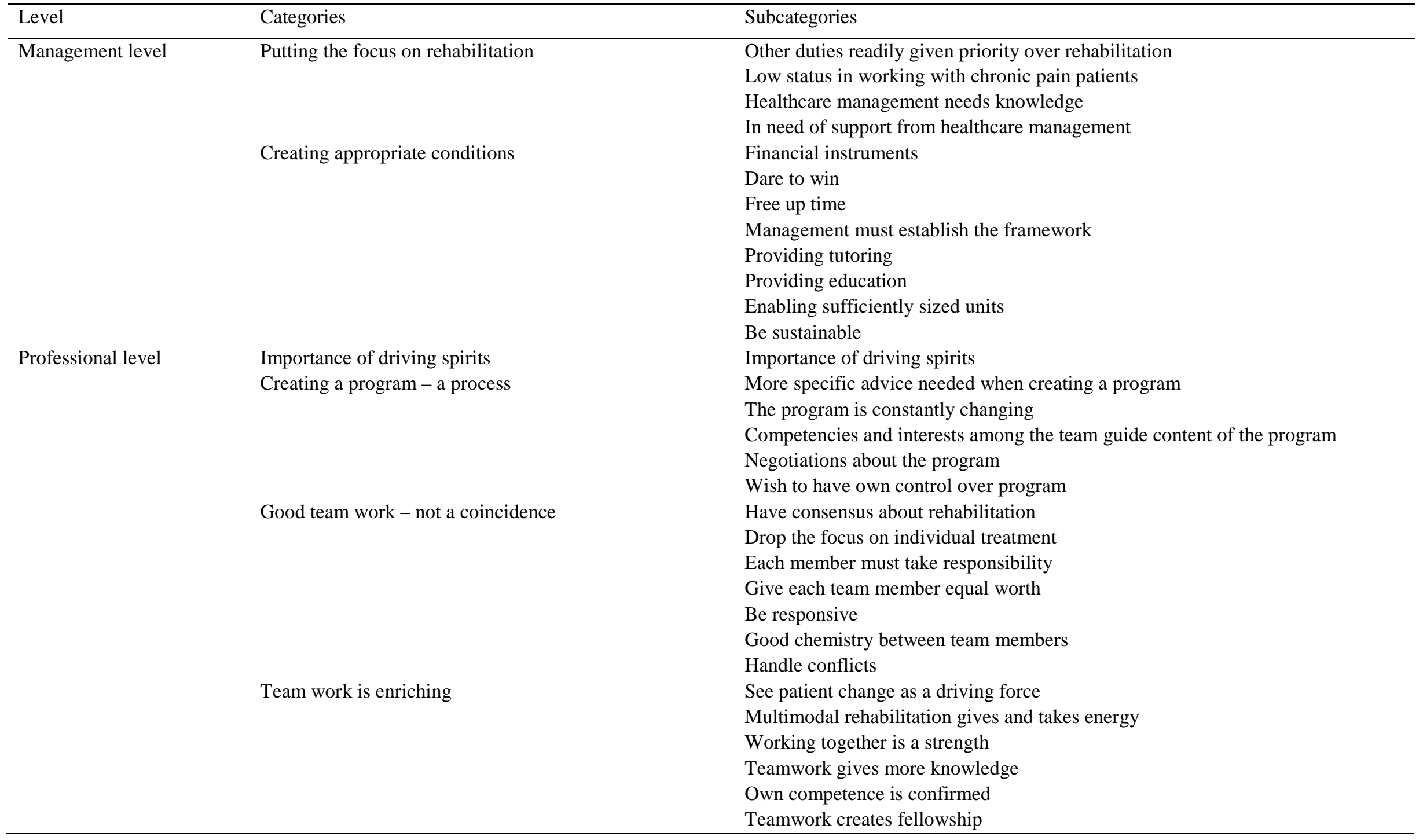


